\title{
Optimization method to suppress background for imaging multiple planes
}

\author{
Vannhu Le \\ Department of Optical Engineering \\ Le Quy Don Technical University, 236 Hoang Quoc Viet Street \\ Tu Liem District, Hanoi, Vietnam \\ State Key Laboratory of Modern Optical Instrumentation \\ College of Optical Science and Engineering \\ Zhejiang University, Hangzhou 310027, P. R. China \\ Institute of Research and Development \\ Duy Tan University, Da Nang 550000, Vietnam \\ levannhu_mta2015@yahoo.com
}

Received 19 March 2018

Accepted 12 December 2018

Published 18 January 2019

\begin{abstract}
3D live imaging is important for better understanding of biological processes. To obtain biological dynamic process, high imaging speed is required. In order to improve speed in 3D live imaging, simultaneous imaging of multiple planes throughout a 3D volume has been proposed. However, a main disadvantage of this method is the cross-talk from neighboring imaging planes. In this paper, we propose an optimization method to suppress background from neighboring imaging planes. A D-aperture is used to generate multiple light sheets. An optimization method to suppress background is presented. The simulation results demonstrated that the proposed method can be used to suppress the effectiveness of background from neighboring light sheets.
\end{abstract}

Keywords: Fluorescence microscopy; imaging systems; supper-resolution.

\section{Introduction}

3D live imaging is important for better understanding of biological processes. For 3D biological imaging, some techniques have been introduced, such as confocal microscopy, wide-filed microscopy and light sheet microscopy. For confocal microscopy, ${ }^{1,2}$ the technique is simple, but it has some limitations including optical aberrations due to mechanical rephrasing, photobleaching and photodamage due to excitation of out-focal planes, and limited temporal resolution. For wide-field microscopy, the technique has high temporal resolution, but the

This is an Open Access article published by World Scientific Publishing Company. It is distributed under the terms of the Creative Commons Attribution 4.0 (CC-BY) License. Further distribution of this work is permitted, provided the original work is properly cited. 
photobleaching and photodamage are main disadvantages. The light sheet microscopy had some advantages such as high temporal resolution, minimal photobleaching and photodamage, and reduced background. $^{3,4}$ Over the past few years, various methods of light sheet microscopy have been developed to improve spatial and temporal resolution. These include thinner light sheet microscopy, ${ }^{5}$ the STED light sheet microscopy ${ }^{6}$ objective coupled planar illumination microscopy ${ }^{7}$ confocal light sheet microscopy, ${ }^{8}$ SIM light sheet microscopy, ${ }^{9}$ multiple light sheet microscopy ${ }^{10}$ individual molecule localization light sheet microcopy, ${ }^{11}$ light sheet microscopy with Bessel beam, ${ }^{12}$ airy beam, ${ }^{13}$ lattice beams ${ }^{14}$ and electric lens, ${ }^{15}$ multiview light sheet microscopy ${ }^{16}$ and so on. In order to obtain biological dynamic process, high imaging speed is required. For light sheet microscopy, there are many variants for the improvement of imaging speed.

In light sheet microscopy, speed depends on the band-width limitations of modern piezoelectric scanners which are used to reposition heavy detection lens quickly. To overcome this problem, several methods have been developed, aiming to avoid the shift of detection lens. In detection arm, some methods have been introduced to extend depth of field, such as spherical aberrations, ${ }^{17}$ the cubic phase mask ${ }^{18}$ or rapidly defocusing of the detected wavefront with an electrically tunable lens, ${ }^{19}$ oblique illumination, ${ }^{20}$ confocally aligned oblique illumination, ${ }^{21}$ or remote focusing through wavefront engineering. ${ }^{22}$ However, these methods sacrifice sensitivity (e.g., due to aberrations, incomplete use of the detection numerical aperture, and inevitably short pixel dwell time) and spatial resolution (usually on the order of a few micros), rendering them incompatible with subcellular structures. ${ }^{23}$

Another major challenge to improve the speed of light sheet microscopy is that two-dimensional (2D) image planes must be serially achieved to generate a $3 \mathrm{D}$ volume. To overcome the limitation of serial image acquisition, some methods have been developed by using multiple light sheets which can enable simultaneous imaging through a $3 \mathrm{D}$ volume. ${ }^{10,23}$ Dean et al. have introduced a method based on illuminating specimen with three light sheets which are arranged the deviation of the in-focus plane and each dependently detected. The method has high light-efficient, high speed and is cross-talk-free. ${ }^{23}$ However, the model of proposed method has a requirement that specimen must be placed tilted and long enough to ensure that the distance between light sheets can remove cross-talk from neighboring light sheets. In addition, the method introduces spherical aberrations in two outside light sheets. Another method has also been suggested to illuminate specimen with multiple light sheets which are arranged at focusing position in the optical axis of detection lens, such as diffraction grating ${ }^{24}$ and Daperture. ${ }^{10}$ This method enables simultaneous imaging through a $3 \mathrm{D}$ volume using diffraction ${ }^{25}$ or refraction optics. ${ }^{26}$ Nevertheless, the fluorescence signal imaged in each plane is reduced by the number of multiple light sheets. A major disadvantage of the method is the high background from cross-talk of neighboring light sheets because the distance between light sheets is too short. In this paper, we propose an optimization method to suppress background which causes by cross-talk from neighboring light sheets. In order to reduce the background from neighboring light sheets, we optimize the value of the numerical aperture of detection lens to obtain the overall point spread function of light sheet microscopy which only allows the area illuminated by the main light sheet to obtain the image on detector. We use D-aperture method for generating multiple light sheets to demonstrate our concept. The D-aperture is a simple method to generate multiple light sheets, low-cost, and can be easily performed without the change of conjugation of the optical system. ${ }^{10}$

\section{Theory for Generating Multiple Light Sheets}

Two fundamental techniques to generate the light sheet have been introduced. The one is using a cylindrical lens to focus an expanded collimated laser beam in one dimension. This is also referred to as a static light sheet beam because the complete sheet of light is formed instantaneously. Due to its simplicity, the static light sheet is very popular. Another technique, the digitally scanned laser light sheet microscopy employs a single, thin laser beam that is quickly scanned by a laser scanner (galvo mirror) over the field of view to generate a sheet of light with even illumination along the same height of the light sheet. This is also referred to as a virtual light sheet beam. In this paper, we use the second method to generate light sheets. The shape of D-aperture is shown in Fig. 1. 


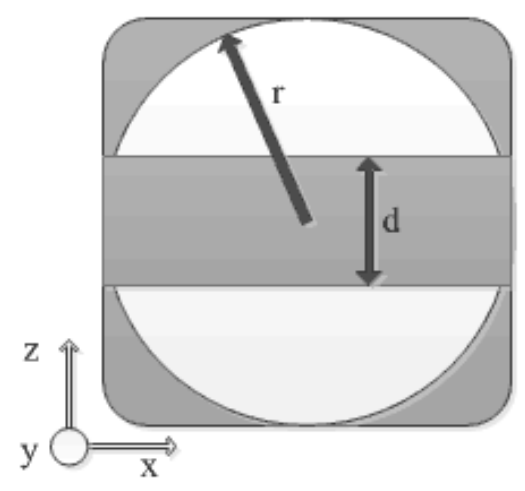

Fig. 1. The D-aperture for the generation of multiple light sheets. The spatial filter is placed at the pupil plane of illumination lens for generation of multiple spots pattern.

The focal effects of incident light propagating through an objective lens can be calculated by use of vectorial diffraction theory. The electric field near the focus can be obtained from the formulae explicitly derived by the Debye integral,

$$
\begin{aligned}
& \mathbf{E}\left(r_{2}, \varphi_{2}, z_{2}\right) \\
& =i C \iint_{\Omega} \sin (\theta) E_{0} A(\theta, \varphi) P \\
& \quad \times e^{i \Delta a(\theta, \varphi)} e^{i k n\left(z_{2} \cos \theta+r_{2} \sin \theta \operatorname{cons}\left(\varphi-\varphi_{2}\right)\right)} d \theta d \varphi,
\end{aligned}
$$

where $\mathbf{E}\left(r_{2}, \varphi_{2}, z_{2}\right)$ is the electric field vector at the point, $\left(r_{2}, \varphi_{2}, z_{2}\right)$ expressed in cylindrical coordinates whose origin, $C$ is the normalized constant, $E_{0}$ is the amplitude function of the input light, $A(\theta, \varphi)$ is a $3 \times 3$ matrix related to the structure of the imaging lens and $P$ is Jonse's vector of the incident light. $\Delta a(\theta, \varphi)$ is the parameter of phase delay generated by the phase plate.

When the objective lens satisfies the sine condition, $A(\theta, \varphi)$ can be presented by

$$
\begin{aligned}
A(\theta, \varphi)= & \sqrt{\cos \theta} \\
& \times\left[\begin{array}{ccc}
1+(\cos \theta-1) \cos ^{2} \varphi & (\cos \theta-1) \cos \varphi \sin \varphi & -\sin \theta \cos \varphi \\
(\cos \theta-1) \cos \varphi \sin \varphi & 1+(\cos \theta-1) \sin ^{2} \varphi & -\sin \theta \sin \varphi \\
\sin \theta \cos \varphi & \sin \theta \sin \varphi & \cos \theta
\end{array}\right] .
\end{aligned}
$$

The overall point spread function of LSM can be calculated by multiplying the PSF of illumination lens and that of detection lens. The overall PSF of LSM system can be presented by

$$
\begin{aligned}
& \operatorname{PSF}_{\text {overall }}(x, y, z) \\
& \quad=\operatorname{PSF}_{\text {Illu }}(x, y, z) \times \operatorname{PSF}_{\text {Detec }}(x, y, z),
\end{aligned}
$$

where $\mathrm{PSF}_{\text {overall }}, \mathrm{PSF}_{\text {Illu }}, \mathrm{PSF}_{\text {Detec }}$ are the overall point spread function, the illumination point spread function and the detection point spread function, respectively.

We show the property of the point spread function of D-aperture placed at the pupil plane of illumination lens. We consider the point spread function of illumination lens for NA equal to 0.3 as an example, the index of medium is 1.33 and the wavelength used is $640 \mathrm{~nm}$. The point spread function for different values of factor $d / r$ is shown in Fig. 2, where the values of factor $d / r$ are set as 0 , $0.4,0.8,1.2$ and 1.6. As shown in Fig. 2, when the value of factor $d / r$ is set as zero, the intensity distribution of the point spread function of Gaussian beam is shown in Fig. 2(a). When the value of factor $d / r$ is not zero, the multiple-spot patterns are generated as shown in Figs. 2(b), 2(c) and 2(d). As can be seen from Fig. 1, when the value of factor $d / r$ is bigger, the reduction of laser power is bigger. This is a main disadvantage of $\mathrm{D}$-aperture technique for the generation of multiple-spots pattern. However, this can be improved upon by using donut shaped beam that has its maximum energy at the pheriphery rather than at the center, as is the case for Gaussian beam. ${ }^{10}$

Based on the point spread functions of illumination lens shown in Fig. 2, light sheets are generated by scanning quickly the point spread functions along $x$-axis. In the mathematical model, the light sheet is obtained by integrating the PSF of thin light beam of the illumination lens, $\operatorname{PSF}(x, y, z)$ along $x$ direction, which can be presented as

$$
\operatorname{PSF}_{\text {Illu }}(y, z)=\int \operatorname{PSF}(x, y, z) d x .
$$

Based on the use of Eq. (4), the multiple light sheets which correspond to multiple-spot patterns of D-aperture are shown in Figs. 3(b)-3(e), while single light sheets of Gaussian beam are depicted in Fig. 3(a). As can be seen from Figs. 3(b)-3(e), it is clear that the number of light sheets increases with the value of factor $d / r$. In addition, the thickness of light sheets reduces. This means that the thickness of light sheets is thinner. In other words, the axial resolution is improved. It is not difficult to find that intensity value of central light sheet is the biggest among all light sheets. When value of factor $d / r$ is increasing, the intensity value of outside light sheets increases and approaches the intensity value of 
V. Le

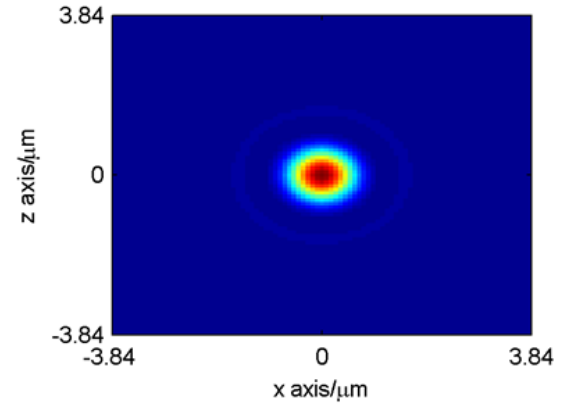

(a)

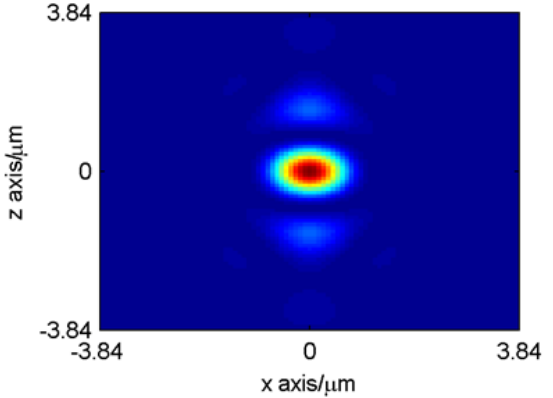

(b)

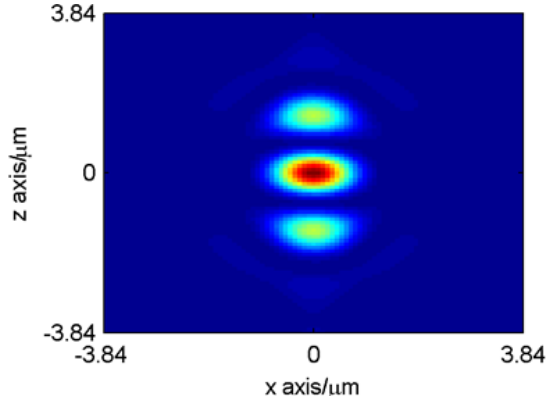

(c)

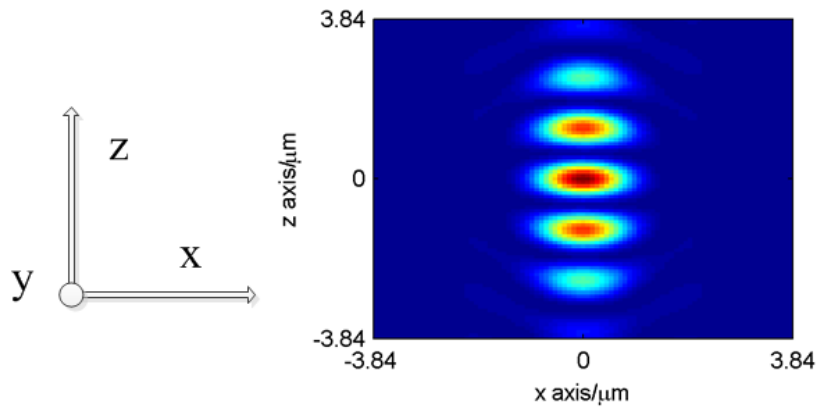

(d)

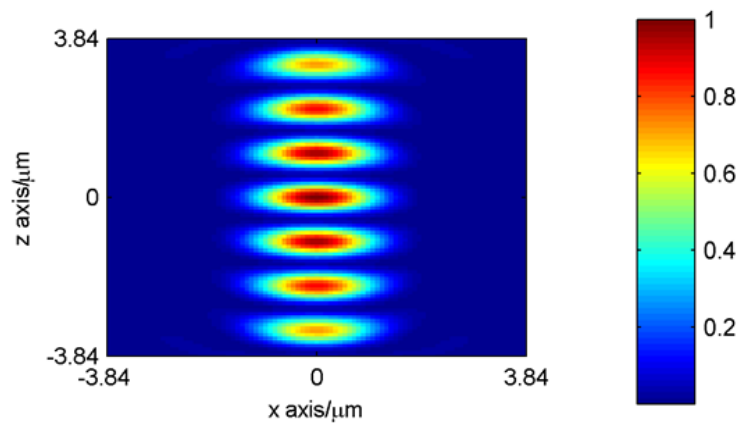

(e)

Fig. 2. (a)-(e) The point spread function of D-aperture for different values of factor $d / r$. The values of factor $d / r$ are set as $0,0.4$, $0.8,1.2$ and 1.6.

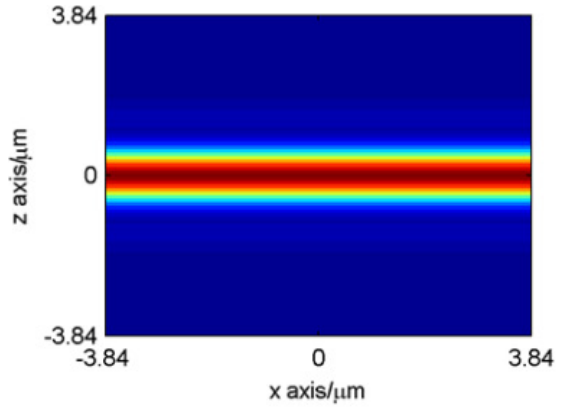

(a)

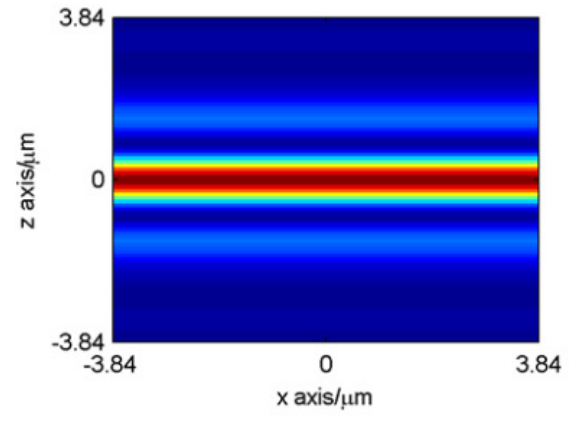

(b)

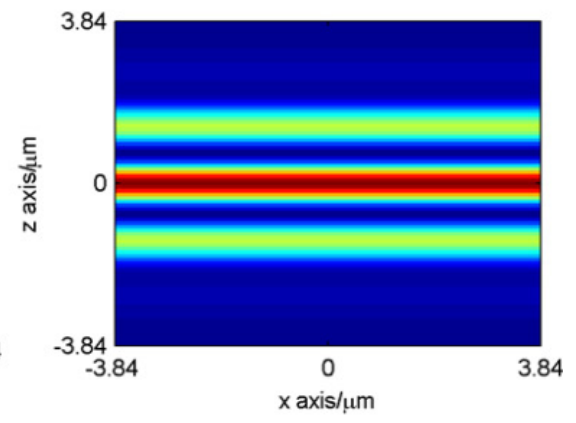

(c)

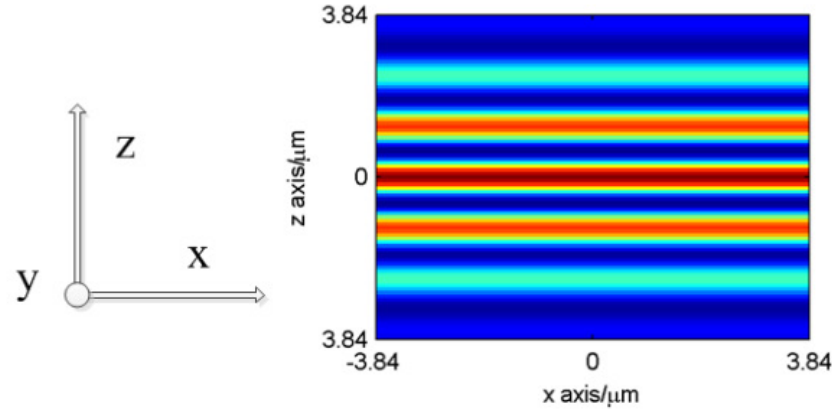

(d)

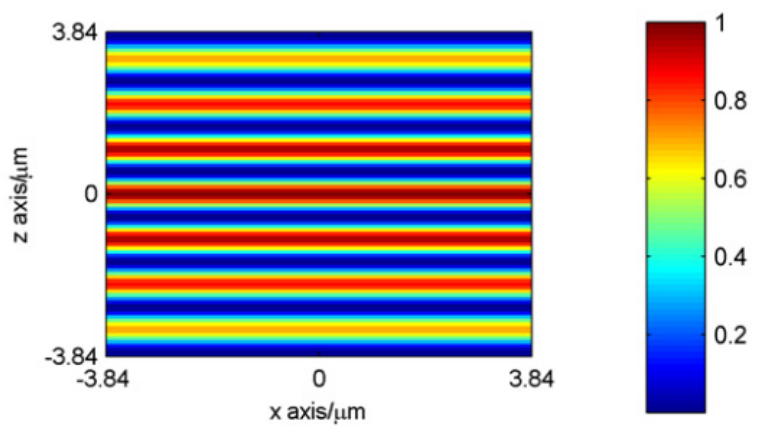

(e)

Fig. 3. (a)-(e) The multiple light sheets of D-aperture combined scanning processing for different factors of $d / r$. The factor of $d / r$ is equal to $0,0.4,0.8,1.2$ and 1.6 . 
central light sheet. Therefore, we should choose suitable value of factor $d / r$ in order to ensure that the imaging quality in all light sheets that are used to obtain image is near.

\section{Optimization to Suppress the Background from Neighboring Imaging Planes}

In order to improve imaging speed, multiple light sheets are used. However, the cross-talk from neighboring light sheets is the main disadvantage. In this paper, the concept to suppress background from neighboring light sheets is introduced. In order to obtain this result, the aim of this work is to find the appropriate numerical aperture of detection lens which can remove the neighboring light sheets in overall point spread function. Therefore, the numerical aperture of detection lens is optimized to satisfy the condition by properly arranging peak intensities of multiple light sheets and intensity distribution of point spread function of detection lens along $z$-axis. The position of peak intensity of light sheet which is used to obtain image coincides with that of the highest peak intensity of point spread function of detection lens, while the positions of peak intensity of neighboring light sheets coincide with that of low intensity of point spread function of detection lens. It notes that the low intensity values of point spread function of detection are nearly zero. As is well known, the overall point spread function is calculated by multiplying both point spread functions of illumination and detection lens, so based on the condition described above, the intensity distributions of neighboring light sheet on overall point spread function are nearly zero. This means that the influence of background which is caused by cross-talk from neighboring light sheets is suppressed.

Based on the multiple light sheets in Fig. 3, in this paper, we use the suitable NA of detection lens to equal 1.1. For this detection lens, the point spread function in $z x$ plane is shown in Fig. 5. Based on the use of NA of detection lens to equal 1.1, the relation between the illumination, detection and overall point spread functions are shown in Fig. 4. In Fig. 4, three $d / r$ values of $1,1.2$ and 1.4 are

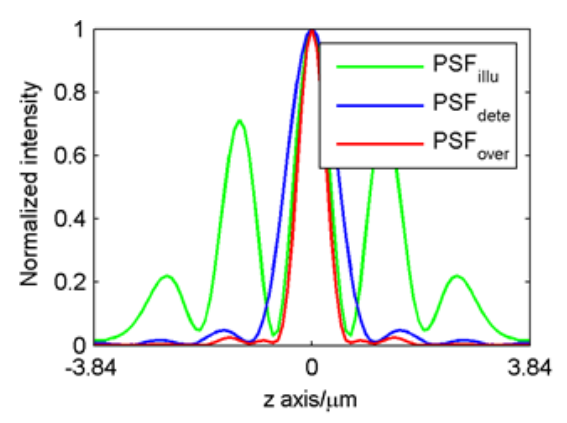

(a)

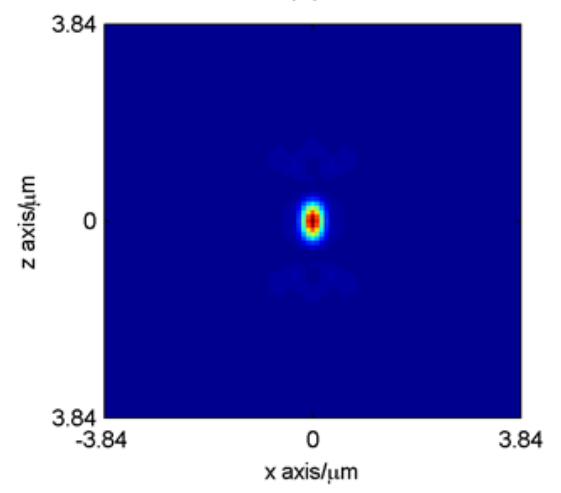

(a1)

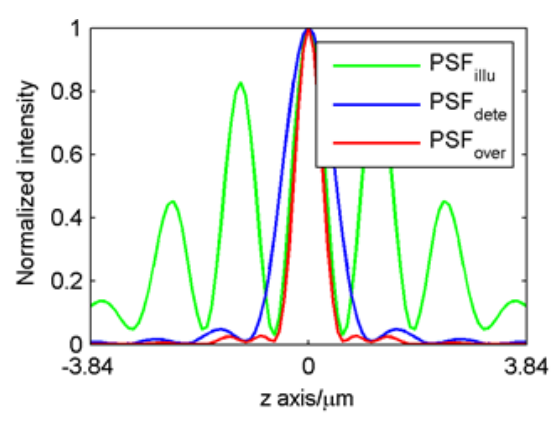

(b)

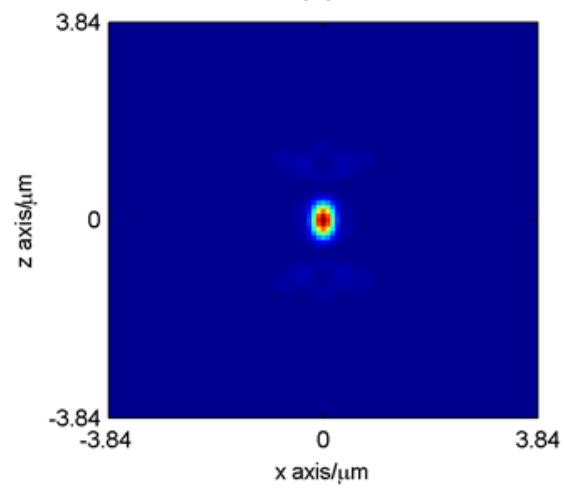

(b1)

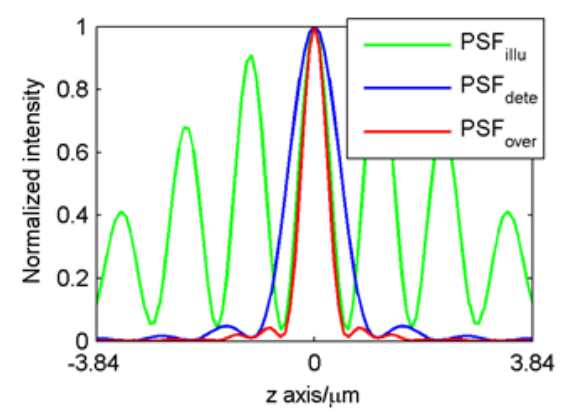

(c)

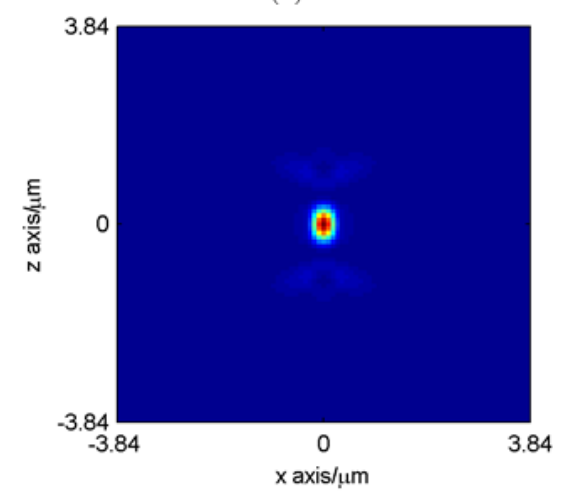

(c1)

Fig. 4. (a), (b) and (c) The illumination, detection and overall point spread functions for the $d / r$ factors of $1,1.2$ and 1.4 along $z$ axis and (a1), (b1) and (c1) The overall point spread functions for $d / r$ factors of $1,1.2$ and 1.6 in $z x$ plane. 


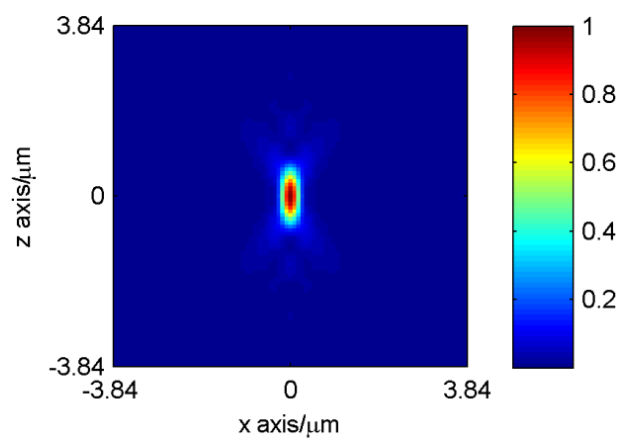

Fig. 5. The point spread function of the detection lens in $z x$ plane.

shown. As Figs. 4(a), 4(b) and 4(c) show, for three $d / r$ values of $1,1.2$ and 1.4 , there are four outside light sheets close to the central light sheet. They have the main effect on the central light sheet. However, it can be seen that the positions of the highest intensity values of the four outside light sheets are arranged at the positions where intensity values of the point spread function of detection lens are close to zero. By multiplying point spread functions of illumination lens for $d / r$ values of $1,1.2$ and 1.4 and detection lens along $z$-axis, the profile of intensity distribution of overall point spread function of light sheet microscopy is shown in Figs. 4(a), 4(b) and 4(c) by the red line. It is clear that the influence of four outside light sheets on image of central light sheet is reduced remarkably. This means that the background caused by crosstalk from neighboring light sheet is suppressed. The $2 \mathrm{D}$ overall point spread functions for $d / r$ values of 1 , 1.2 and 1. 4 are shown in Figs. 4(a1), 4(b1) and $4(\mathrm{c} 1)$, respectively. As these figures depict, a solid lobe of overall point spread function is shown, while outside intensity value is very small. This means that the influence of neighboring light sheet on the light sheet, which is used to obtain imaging data, is reduced effectively. As can be seen for the overall point spread functions in Fig. 4, the reduction of background from neighboring light sheets for the $d / r$ values of $1,1.2$ and 1.4 is nearly the same because the change between light sheets of multilight sheet is slow when the $d / r$ value varies. In this paper, we adopted the $d / r$ value of 1.4 to consider the effectiveness of other light sheets.

For the $d / r$ value of 1.4 , the point spread function of illumination lens is shown in Fig. 6(a), while the multi-light sheets are shown in Fig. 6(b). The illumination, detection and overall point spread function along $z$ axis are shown in Fig. 7. For the multiple light sheets of $d / r$ value of 1.4 , we additionally consider the effect of background of two outside light sheets from neighbor light sheets. The intensity distribution of point spread function is shown by the blue lines in Fig. 7. Figure 7 shows the reason why cross-talk from neighboring light sheets are suppressed. For central light sheet, there are four light sheets close to it. As Fig. 7(a) shows, the positions of highest intensity values of the four outside light sheets are arranged at the same position where intensity values of the point spread function of detection lens is close to zero. By multiplying point spread functions of illumination and detection lens along $z$ axis, the profile of intensity distribution of overall point spread function of light sheet microscopy is shown in Fig. 7(a) by the red line. It is clear that the influence of four outside light sheets on image of the central light sheet is reduced remarkably. This means that the background caused by cross-talk from neighboring light sheet is suppressed. As Fig. 7(b) and 7(c) show, it is

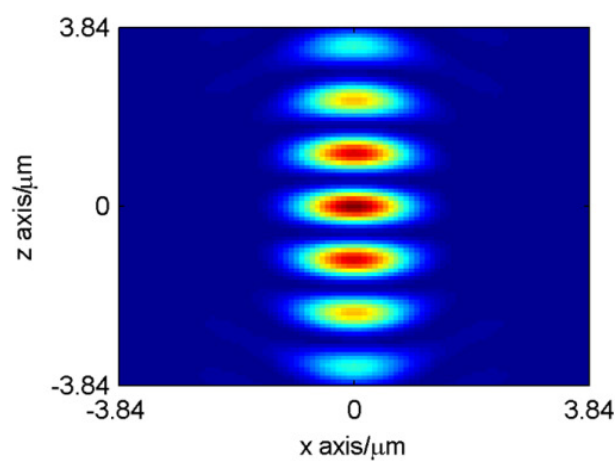

(a)

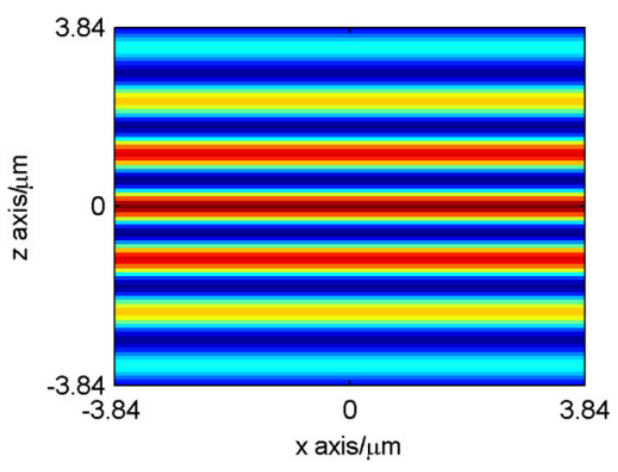

(b)

Fig. 6. The intensity distribution at the $d / r$ value of 0.7 . (a) shows the point spread function and (b) shows multiple light sheets. 


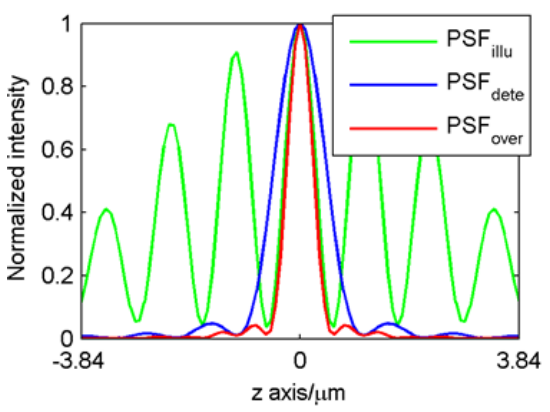

(a)

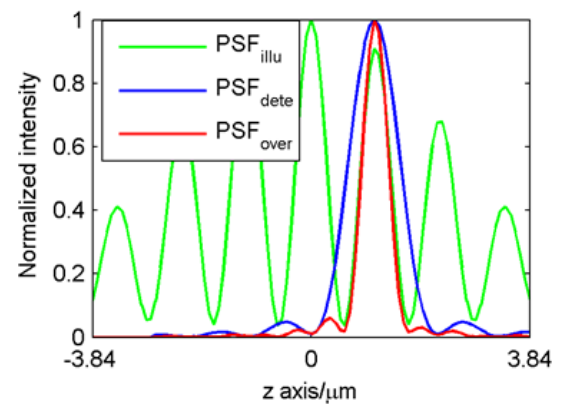

(b)

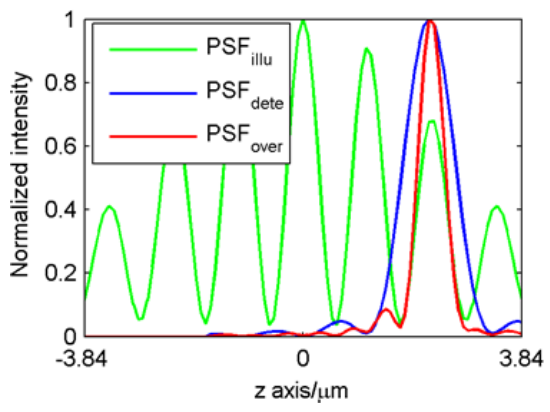

(c)

Fig. 7. The condition to suppress background from neighboring light sheets. PSF illu $_{\text {is }}$ the point spread function of illumination lens; $\mathrm{PSF}_{\text {dete }}$ is the point spread function of detection lens; $\mathrm{PSF}_{\text {over }}$ is the overall point spread function.

not difficult to see that other light sheets has imaging performance similar to the central light sheet. This means that the cross-talk from its neighboring light sheet suppressed when these light sheets are used to obtain image. The $2 \mathrm{D}$ overall point spread functions of three light sheets are shown in Fig. 8. As Fig. 8 depicts, a solid lobe of overall point spread function of each light sheets is shown, while the outside intensity value is very small. This means that the influence of neighboring light sheet on the light sheets, which is used to obtain imaging data, is reduced effectively.

In order to indicate the effectiveness of the proposed method in outside focal plane, we research imaging property at outside focal plane. Figure 9(a) shows the intensity distribution of point spread function of illumination lens in $z y$ plane. It can be seen that the intensity peaks of the five light sheets are kept at the same positions along $y$-axis. This means that our concept can be obtained better at outside focal plane. First, we determine the field of view of multiple light sheets. In order to determine the field of view, the intensity distributions of Gaussian light sheet and multiple light sheets on $z$-axis are shown in Fig. 9(b). We use the intensity level equal to 0.5 to determine the field of view. As Fig. 9(b) shows, it is not difficult to see that the field of view of multiple light sheets is bigger than that of Gaussian light sheet. The field of view of multiple light sheets ranges from $-12.5 \mu \mathrm{m}$ to $12.5 \mu \mathrm{m}$. The normalized intensity distribution of multi-focus pattern in $x z$ plane at the position of $y=12.5 \mu \mathrm{m}$ is shown in Fig. 9(c). As Figs. 6(a) and 9(c) show, the normalized intensity distributions of multi-focus pattern are near the same. This means that the performance of multi-light sheets at the position of $y=12.5 \mu \mathrm{m}$ is nearly the same as the multi-light sheets. The multi-light sheets at the position of $y=12.5 \mu \mathrm{m}$ are shown in Fig. 9(d). As Figs. 9(d) and $6(\mathrm{~b})$ show, the normalized intensity distribution of multi-light sheets at two positions are nearly the same and therefore the optimal condition presented above is still kept at the position. The intensity distribution of multi-light sheets at the

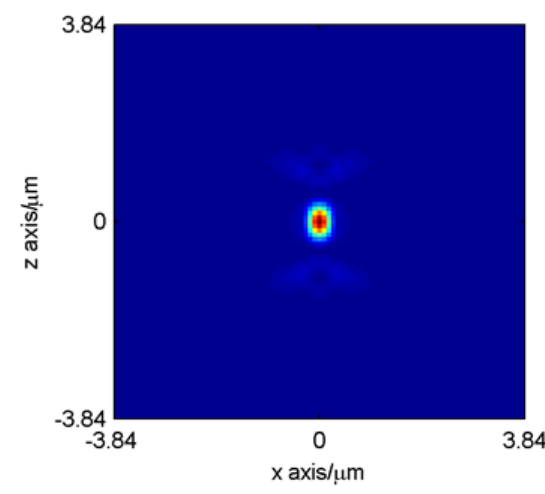

(a)

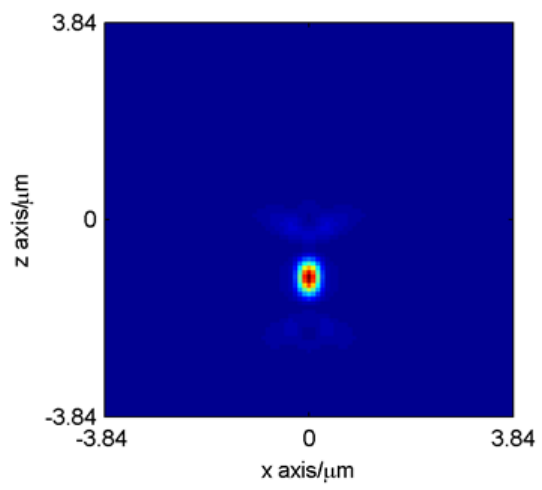

(b)

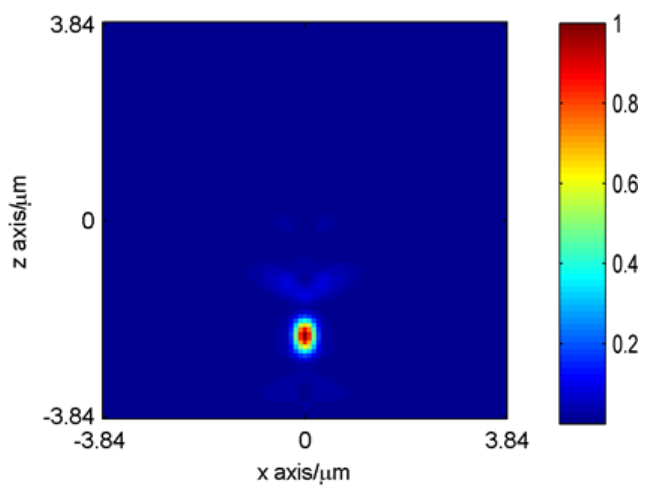

(c)

Fig. 8. The overall point spread function of center light sheet in $x, z$ plane. 


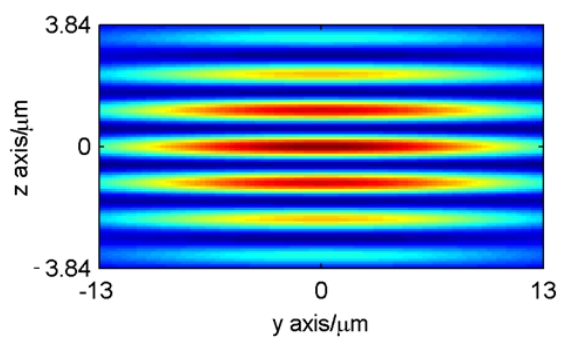

(a)

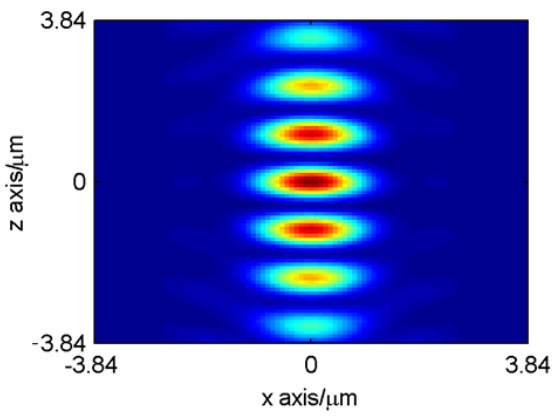

(c)

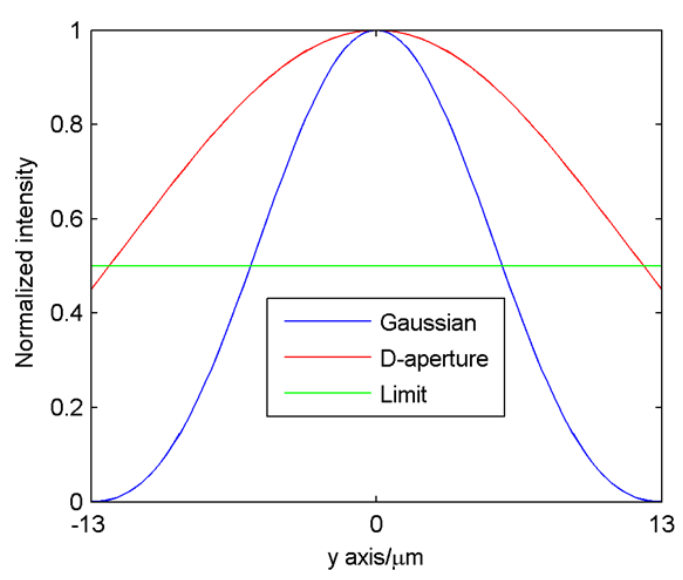

(b)

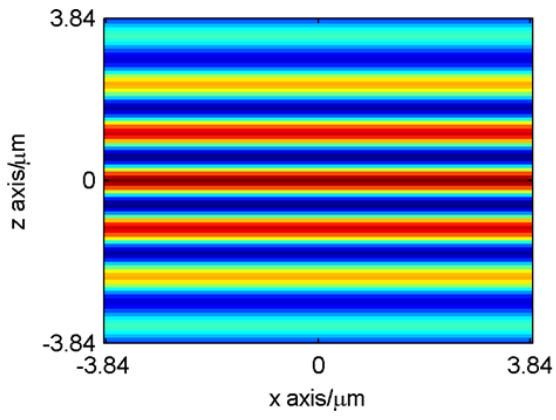

(d)

Fig. 9. Intensity distributions. (a) shows the intensity distribution in yz plane, (b) shows the intensity distributions of Gaussian light sheet and multi-light sheets along $y$-axis, (c) shows the intensity distribution in $z x$ plane at outside position and (d) shows multi light sheets.

position of $y=12.5 \mu \mathrm{m}$ along $z$ axis and in $z x$ plane are shown in Figs. 10 and 11. As Figs. 10 and 11 show, the influence of neighboring light sheet to the light sheets, which is used to obtain imaging data, is reduced effectively.
Here, we show the imaging performance of Gaussian light sheet. Figure 12 shows the intensity distribution of Gaussian light sheet. Figure 12(b) shows the illumination, detection and overall point spread function. Figure 12(a) shows the overall

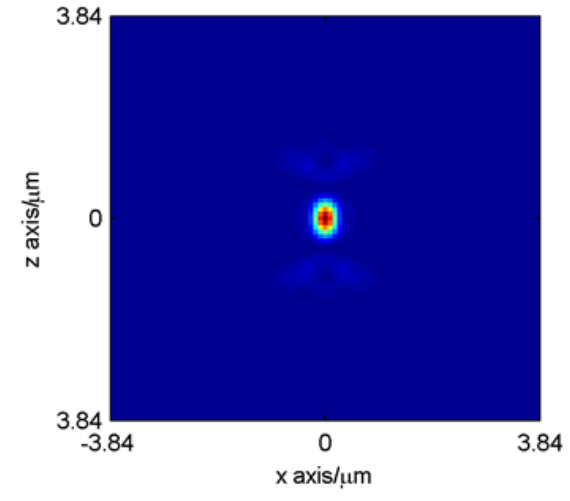

(a)

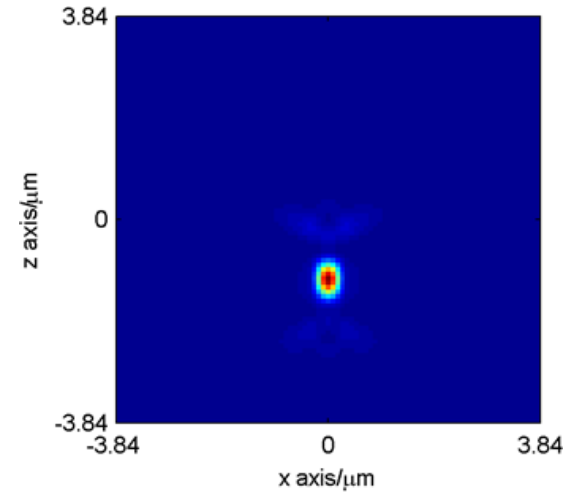

(b)

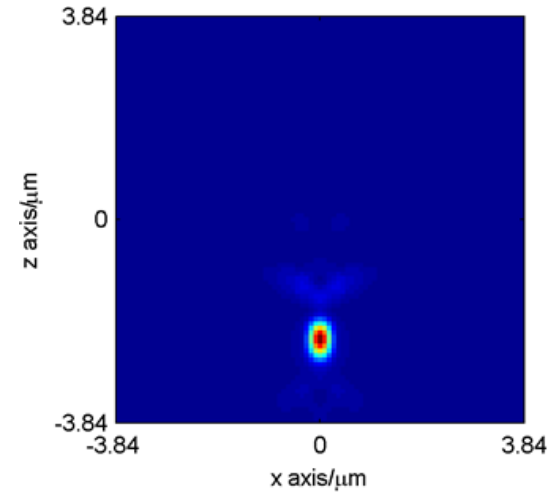

(c)

Fig. 10. The condition to suppress background from neighboring light sheets. PSF illu $_{\text {is }}$ the point spread function of illumination lens; $\mathrm{PSF}_{\text {dete }}$ is the point spread function of detection lens; $\mathrm{PSF}_{\text {over }}$ is the overall point spread function. 


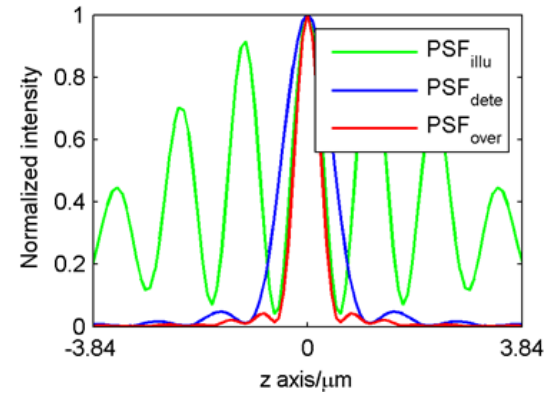

(a)

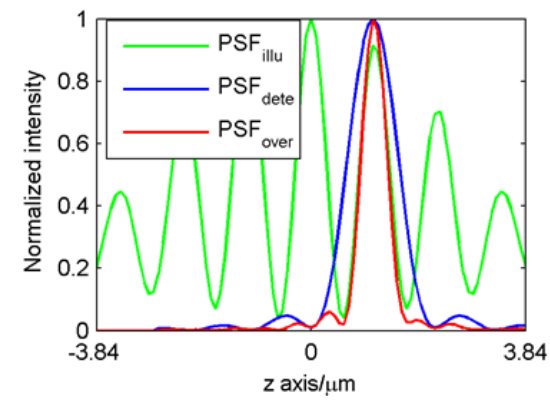

(b)

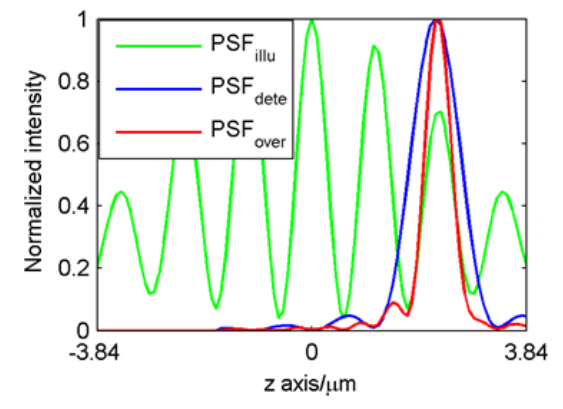

(c)

Fig. 11. The overall point spread function of light sheets in $x z$ plane.

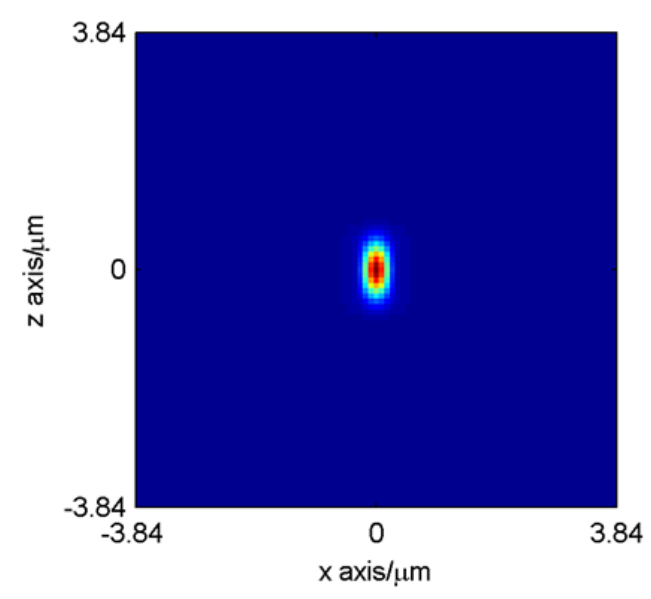

(a)

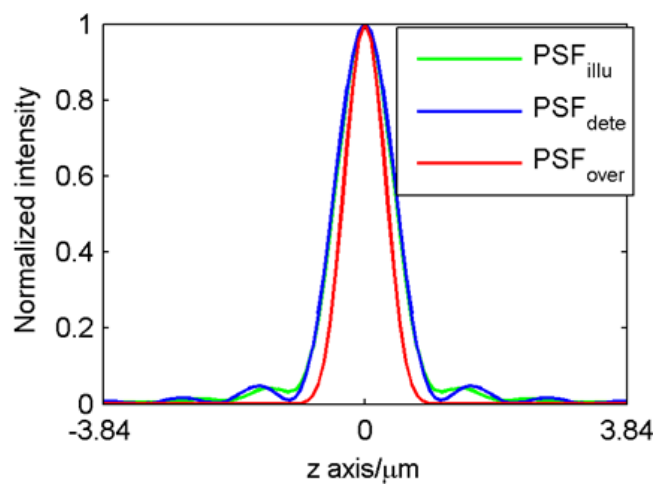

(b)

Fig. 12. The intensity distribution of Gaussian light sheet.

point spread function in the $z x$ plane. Based on the use of Figs. 4(c) and 12(b), the full width half maximum (FWHM) of multi-light sheets is smaller about 1.3 times than that of Gaussian light sheet.

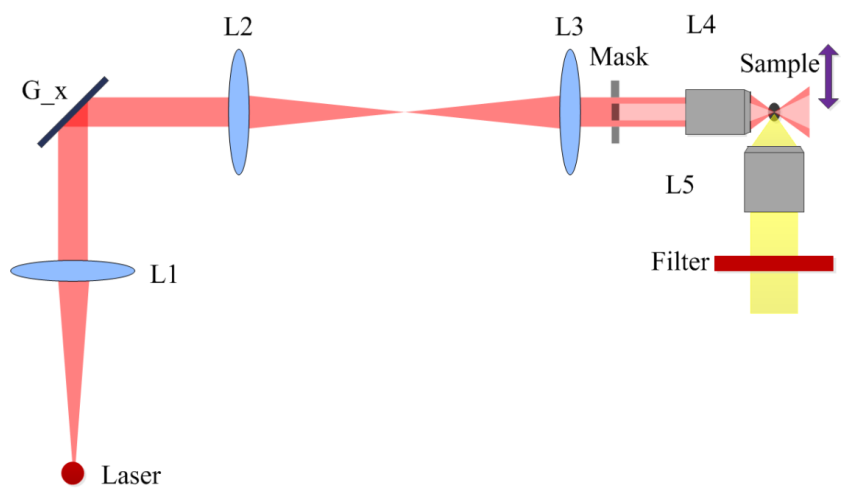

Fig. 13. Conjugation of imaging system. L1, L2, L3 are the lenses; L4 is the illumination lens; L5 is the detection lens. The shape of D-aperture mask is shown in Fig. 1.
The conjugation of imaging system of the illumination part is shown in Fig. 12. The G-x galvo mirror is placed at the position which conjugates with the pupil plane of the illumination lens. G_x, is used to generate the light sheet. Sample is moved along $z$ axis to obtain each stack. We use the sample moves, so the position of the mask can be placed after L1 lens or L3 lens. In this conjugation of imaging system, the mask position is placed after L3 lens. The design of the mask is shown in Fig. 1. The mask is simple, it can be easy manufactured. For the detection part, in order to be simultaneously detected, several optical strategies have been performed that enable simultaneous imaging multiple planes throughout a $3 \mathrm{D}$ volume using refractive ${ }^{25}$ or diffractive optics. ${ }^{26,27}$ Nevertheless, in each case, the fluorescence signal imaged in each plane is reduced by the degree of parallelization.

As is well known, when the NA of the illumination lens is smaller, field of view of light sheet is 
increasing. In order to obtain the high penetration depth, we use the small NA of the illumination lens. For the given $d / r$ value, the smaller NA of the illumination lens, the distance between light sheets is bigger. This means that we should choose the smaller NA of the detection lens in order to remove the background from neighboring light sheets.

\section{Conclusion}

In this paper, we have proposed an optimization method to suppress background in imaging planes. The condition for choosing the numerical aperture of detection lens is shown. The multiple light sheets are generated by using the D-aperture with scanning processing. We have also presented an evaluation approach based on the use of the point spread function. The simulation result demonstrates that the proposed method can be used to suppress cross-talk from neighboring light sheets. In the future, the experimental results will be acquired.

\section{Conflict of Interest}

The authors declare that there are no conflicts of interest related to this article.

\section{Acknowledgments}

This work is supported by the Vietnam National Foundation for Science and Technology Development (NAFOSTED) under Grant number (103.03-2018.08).

\section{References}

1. P. Gao, B. Prunsche, L. Zhou, K. Nienhaus, G. U. Nienhaus, "Background suppression in fluorescence nanoscopy with stimulated emission double depletion," Nat. Photonic. 11, 163-169 (2017).

2. B. Huang, W. Wang, M. Bates, X. Zhuang, "Threedimensional super-resolution imaging by stochastic optical reconstruction microscopy," Science 319, 810-813 (2008).

3. B. Richards, E. Wolf, "Electromagnetic diffraction in optical systems. Structure of the image field in an aplanatic system," Proc. R. Soc. A 253, 358-379 (1959).

4. R. Loic, C. L. William, K. C. Raghav, W. Yinan, C. Michael, W. M. Eugene, J. K. Philipp, "Adaptive light sheet microscopy for long-term, high resolution imaging in living organisms," Nat. Biotechnol. 12, 1267-1281 (2016).
5. E. Fuchs, J. S. Jaffe, "Thin laser light sheet microscopy for microbial oceanography," Opt. Exp. 10(2), 145 (2002).

6. M. Friedrich, Q. Gan, V. Ermolayev, G. S. Harms, "STED-SPIM: Stimulated emission depletion improves sheet illumination microscopy resolution," Biophys. J. 100(8), L43-L45 (2011).

7. T. F. Holekamp, D. Turaga, T. E. Holy, "Fast threedimensional fluorescence imaging of activity in neural populations by objective coupled planar illumination microscopy," Neuron. 57(5), 661-672 (2008).

8. L. Silvestri, A. Bria, L. Sacconi, G. Lannello, F. S. Pavone, "Confocal light sheet microscopy: Micronscale neuroanatomy of entire mouse brain," Opt. Exp. 18, 20482-20598 (2012).

9. R. Itoh, J. R. Landry, S. Hamann, O. Solgaard, "Light sheet fluorescence microscopy using highspeed structured and pivoting illumination," Opt. Lett. 41(21), 5015-5018 (2016).

10. K. Mohan, S. B. Purnapatra, P. P. MOndal, "Three dimensional fluorescence imaging using multiple light sheet microscopy," Plos 39, 4715 (2014).

11. A. K. Gustavsson, P. N. Petrov, M. Y. Lee, Y. Shechtman, W. E. Moerner, "3D single-molecule super-resolution miecroscopy with a tilted light sheet," Nature Communications 9, 123 (2018).

12. L. Gao, L. Shao, B. C. Chen, E. Betzig, "3D live fluorescence imaging of cellular dynamic using Bessel beam plane illumination microscopy," Protocol. 9(5), 1083 (2014).

13. B. C. Chen, W. R. Legant, K. Wang, L. Shao, D. E. Milkie, M. W. Davidson, C. Janetopoulos, X. S. Wu, J. A. Hammer III, Z. Liu, B. P. English, Y. MimoriKiyosue, D. P. Romero, A. T. Riter, J. LippincottSchwartz, L. Firtz-Laylin, R. D. Mullins, D. M. Mitchell, J. N. Bembenek, A. C. Reymann, R. Bohme, S. W. Grill, J. T. Wang, G. Seydoux, U. S. Tulu, D. P. Kiehart, E. Betzig, "Lattice light-sheet microscopy: Imaging molecules to embryos at high spatiotemporal resolution," Science 346, 1257998 (2014).

14. T. Vettenburg, H. I. C. Dalgarno, J. Nylk, C. CollLlado, D. E. K. Ferrier, T. Czmar, F. J. GunnMoore, K. Dholakia, "Light sheet microscopy using an Airy beam," Nat. Methods 11, 541-544 (2014).

15. M. Duocastella, C. B. Arnold, J. Puchalla, "Selectable light-sheet uniformity using tuned a xial scanning," Microsc. Res. Tech. 80, 250-259 (2017).

16. U. Krzic, S. Gunther, T. E. Saunders, S. J. Streichan, L. Hufnagel, "Multiview light sheet microscopy for rapid in toto imaging," Nat. Meth. 9(7), 730 (2012).

17. R. Tomer, M. Lovett-Barron, I. Kauvar, A. Andalman, V. M. Burns, S. Sankaran, L. Grosenick, M. 
Broxton, S. Yang, K. Deisseroth, "SPED light sheet microscopy: Fast mapping of biological system structure and function," Cell 163, 1796 (2015).

18. O. E. Olarte, J. Andlla, D. Artigas, P. Loza-Alvares, "Decoupled illumination detection in light sheet microscopy for fast volumetric imaging," Optica 2(8), 702 (2015).

19. F. O. Fahrbach, F. F. Voigt, B. Schmid, F. Helmchen, J. Huisken, "Rapid 3D light-sheet microscopy with a tunable lens," Opt. Exp. 21, 21010-21026 (2013).

20. S. Kumar, D. Wilding, M. B. Sikkel, A. R. Lyon, K. T. Macleod, C. Dunsby, "High-speed 2D and 3D fluorescence microscopy of cardiac myocytes," Opt. Exp. 19, 13839 (2011).

21. M. B. Bouchard, V. Voleti, C. S. Mendes, C. Lacefield, W. B. Grueber, R. S. Mann, R. M. Bruno, E. M. C. Hillman, "Swept confocally-aligned planar excitation (SCAPE) microscopy for high-speed volumetric imaging of behaving organisms," Nat. Photonics 9, 113 (2015).

22. E. J. Bothcherby, C. W. Smith, M. M. Kohl, D. Debarre, M. J. Booth, R. Juskaitis, O. Paulsen, T. Wilson "Aberration-free three-dimensional multiphoton imaging of neuronal activity at $\mathrm{kHz}$ rates," Proc. Natl. Acad. Sci. USA 109, 2919 (2012).
23. K. M. Dean, P. Roudot, E. S. Welf, T. Pohlkamp, G. Garrelts, J. Herz, R. Folka, "Imaging subcellular dynamics with fast and light-efficient volumetrically parallelized microscopy," Optica 4(2), 263 (2017).

24. P. P. Mondal, S. Dilipkumar, K. Mohan, "Efficient generation of diffraction-limited multi-sheet pattern for biological imaging," Opt. Lett. 40(4), 609 (2015).

25. S. Geissbuehler, A. Sharipov, A. Godinat, N. L. Bocchio, P. A. Sandoz, A. Huss, N. A. Jensen, S. Jakobs, J. Enderlein, F. Gisou van der Goot, E. A. Dubikovskaya, T. Lasser, M. Leutenegger, "Live-cell mutiplane three-dimensional superresolution optical fluctuation imaging," Nat. Commun. 5, 5830 (2014).

26. Q. Ma, B. Khademhosseinieh, E. Huang, H. Qian, M. A. Bakowski, E. R. Troemel, Z. Liu, "Threedimensional fluorescent microscopy via simultaneous illumination and detection at multiple planes," Sci. Rep. 6, 31445 (2016).

27. B. Hajj, J. Wisniewski, M. EI Beheiry, J. Chen, A. Revyakin, C. Wu, M. Dahan, "Whole-cell, muiticolor superresolution imaging using mutifocus microscopy," Proc. Natl. Acad. Sci. USA 11, 17480 (2014). 